\title{
Vehicle stability criterion based on three-fold line method
}

\author{
Song Shixin ${ }^{(1)}$, Sun Wanchen(2), Xiao Feng(2), Peng Silun(2), Sun Farong ${ }^{(2)}$, Wang Da ${ }^{(2)}$ \\ (1) College of Mechanical and Engineering, Jilin University, Changchun 130022 \\ (2) State Key Laboratory of Automotive Simulation and Control, Jilin University, Changchun 130022 \\ e-mail: xiaofeng4206@126.com
}

\section{SUMMARY}

Stable boundary is analysed and corresponding stability criterion is proposed based on sideslip angle speed- sideslip angle phase plane. First, we analyse the impact of adhesion coefficient, longitudinal speed and front wheel angle on phase plane stable boundary, then we simplify the hyperbolic boundary with polyline. Stability criterion is then built based on the distance between locus and stable boundary. The proposed stability criterion is integrated to vehicle stability control system, and simulations are run under Matlab/Simulink-Carsim co-simulation platform. The results show that stability criterion based on sideslip angle speed-sideslip angle phase plane can evaluate vehicle stability state; under SWD/SIS steering condition and DLC condition, stability control system based on the proposed stability criterion improved vehicle stability significantly.

KEY WORDS: automotive engineering; phase plane method; stability criterion; fold line method.

\section{INTRODUCTION}

Steering sideslip and caused side impact are the main reasons for serious injuries and vehicle collision [1, 2]. In order to improve steering safety and controllability, lots of manufacturers developed electronic stability control system, such as Bosch's ESP [3] system, Toyota's VSC system, etc. When developing such products, developers need to know whether vehicle stability criterion directly determines the control effect of the controller. From this point, it is very important to establish vehicle stability criterion.

Phase plane method is a graphic method used to explain first order \& second order linear system or non-linear system. It is used for analysing system stability, also used for development of stability control system. From the perspective of energy, Guo [4] and Luo and Lai [5] established energy phase plane, analysed the proportional relation between longitudinal kinetic energy and lateral kinetic energy. Pacejka [6], Inagaki et al [7], Sacks and Singh [8], etc. established the phase plane by using the variables - different vehicles' states on the coordinate axis and then conducted the stability analysis. In this process, they used yaw 
rate - sideslip angle phase plane, front axle sideslip angle - rear axle sideslip angle phase plane, sideslip angle - sideslip angle speed phase plane, etc. to show and research vehicle stability. Inagaki thinks that $d \beta-\beta$ phase plane is more suitable for the analysis of vehicle stability. Yamamoto et al [9] and Zhang et al [10] proposed a "two-line method" to represent the stable boundary for the $d \beta-\beta$ phase plane and provided the boundary formula. Yu et al [11] completed the two-line method with the yaw angular velocity method and established the stability criterion. Xiong [12] and Liu [13] introduced the diamond stability criterion. In the stability criterion based on the two-line method, the stability region and the actual stability region are reduced to a certain extent, and the evaluation is relatively conventional.

In this paper, $d \beta-\beta$ phase plane is used as a tool to analyse the impact of different factors on the stable boundary of phase plane; due to the deficiency of the stability criterion of 'double-line method', 'three folded line method' is proposed in order to simplify hyperbolic stable boundary and construct corresponding stability criterion; simulations of stability criterion are run on Simulink-Carsim co-simulation platform.

\section{VEHICLE MODEL}

Vehicle model with two degrees of freedom is constructed to make stability analysis, meanwhile, Dugoff tire model [14] is introduced to calculate wheel's lateral force. Vehicle model is shown in Figure 1. In Figure 1, $O$ is instantaneous center when a vehicle turns; $\delta$ is front wheel angle; $\psi$ is vehicle's actual yaw angle; $\psi_{\text {des }}$ is the ideal yaw angle determined by the actual yaw angle, path curvature and speed; $\alpha_{f}$ is front wheel sideslip angle and $\alpha_{r}$ is rear wheel sideslip angle.

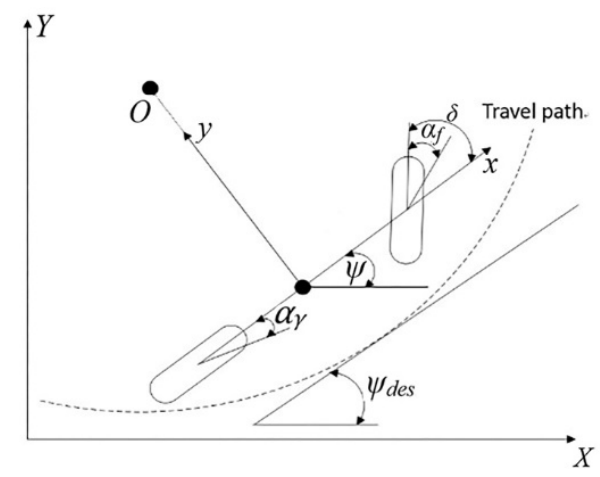

Fig. 1 Vehicle model with two degrees of freedom

Vehicle's lateral displacement $-y$ and yaw angle $-\psi$ are generalized variables. Vehicle motion model with two degrees of freedom can be expressed as:

$$
\begin{aligned}
& m\left(\ddot{y}+V_{x} \dot{\psi}\right)=F_{y f}+F_{y r} \\
& I_{z} \ddot{\psi}=L_{f} F_{y f}-L_{r} F_{y r}
\end{aligned}
$$

In this equation, $m$ is vehicle's mass; $V_{x}$ is longitudinal speed; $F_{y f}$ is front wheel lateral force and $F_{y r}$ is rear wheel lateral force; $I_{z}$ is the moment of inertia; $L_{f}$ is the distance between front axis and vehicle's center of mass; $L_{r}$ is the distance between rear axis and vehicle's center of mass.

Vehicle sideslip angle $\beta$ is approximately expressed as follows: 


$$
\beta=\frac{\dot{y}}{V_{X}}
$$

and introducing it into the Eq. (1) the two degrees of freedom differential equation is derived:

$$
\begin{gathered}
\dot{\beta}=-\dot{\psi}+\frac{F_{y f}+F_{y r}}{m V_{x}} \\
\ddot{\psi}=\frac{L_{f} F_{y f}-L_{r} F_{y r}}{I_{z}}
\end{gathered}
$$

In Equation (3), lateral force is calculated based on the Dugoff tire model [15,16. Model parameters are shown in Table 1:

Table 1. Model parameters

\begin{tabular}{c|r}
\hline The whole vehicle equipment mass $\mathrm{m} / \mathrm{kg}$ & 1350 \\
Wheelbase $/ \mathrm{mm}$ & 2675 \\
Track width $/ \mathrm{mm}$ & 1550 \\
The height of mass center $/ \mathrm{m}$ & 0.4 \\
Tire & $195 / 65 R 15$ \\
Tire rolling radius $/ \mathrm{m}$ & 0.311 \\
Tire Cornering Stiffness $C / \mathrm{kN} / \mathrm{rad}$ & -50000 \\
\hline
\end{tabular}

\section{STABILITY BOUNDARY ANALYZING}

Firstly, vehicle's stability area should be determined according to phase plane analysis method by taking vehicle sideslip angle as the abscissa and taking vehicle sideslip angle speed as the ordinate to construct $d \beta-\beta$ phase plane, as shown in Figure 2. From this figure, it can be seen that if the absolute values of vehicle sideslip angle and the speed are low, then the locus will converge to 0 after some time. At this moment, vehicle's motion state is stable. If absolute values of the vehicle sideslip angle and the speed are high, the locus will move toward the direction of increasing vehicle sideslip angle. At this moment, vehicle is in an unstable state. According to the above criterion, two curves in the first and third quadrants divide the phase figure into the stable area and unstable area. In the stable area, phase trajectory from any initial point will eventually converge to zero, that is, vehicle returns to a stable equilibrium state. Outside the stable area, the motion of the phase trajectory is divergent, vehicle sideslip angle increases sharply and the vehicle is unstable.

Figure 3 shows changes in $d \beta-\beta$ phase plane with the increase of turning angle. In the case of constant speed and adhesion coefficient, if steering wheel's turning angle increases, the stability boundary will change from BL1 to BL2 and BL3. This suggests that the bigger the turning angle is, the more unstable area is. With the increase of speed and pavement adhesion coefficient, the boundary of stable and unstable regions changes. 


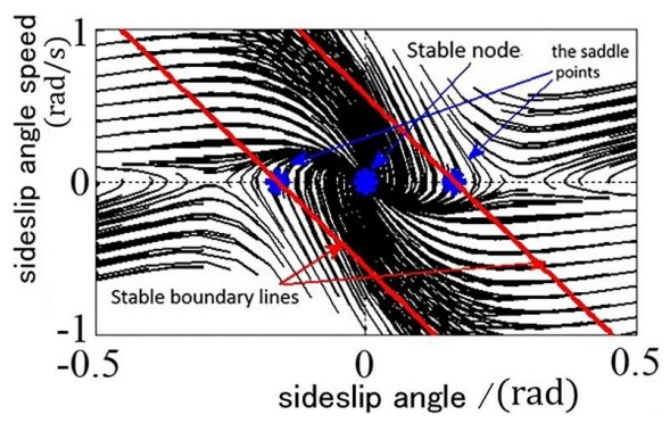

Fig. $2 d \beta-\beta$ phase plane and stable boundary

The stable boundary determined with "double-line method" is represented with a red line in Figure 2. The following inequality is used to express it, [17]:

$$
\left|\beta+B_{1} d \beta\right| \leq B_{2}
$$

In Equation (4), $B_{1}$ and $B_{2}$ are constant coefficients. The intersection of two lines with the $\beta$ axis is the saddle point on both sides of the phase diagram. The slope of the line is:

$$
k_{i}=\frac{\Delta d \beta_{i}}{\Delta \beta_{i}}, i=1,2
$$

In Equation (5), $k$ is the slope of the boundary line.

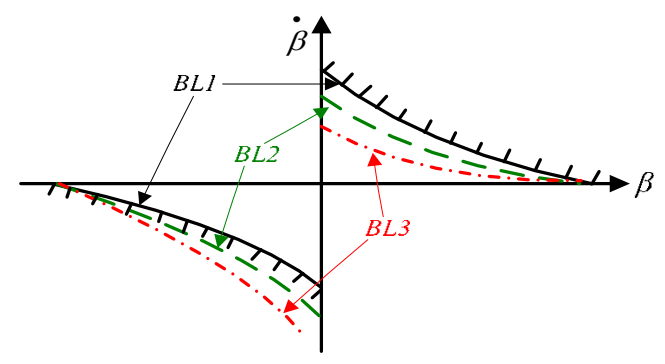

Fig. 3 Stable boundary change with conditions

Based on the following conditions and variables, the stable boundary is analysed.

Table 2. Variable range and condition analysis

\begin{tabular}{crrr}
\hline Variable & Condition & Range & Interval \\
\hline Pavement adhesion coefficient $\mu$ & $v=20 \mathrm{~m} / \mathrm{s}$ & {$[0.1,1]$} & 0.1 \\
Longitudinal speed $v(\mathrm{~m} / \mathrm{s})$ & $\mu=0.8$ & {$[5,40]$} & 5 \\
Front wheel turning angle $\delta(\mathrm{rad})$ & $\mu=0.8, v=20 \mathrm{~m} / \mathrm{s}$ & {$[0,20]$} & 2 \\
\hline
\end{tabular}

Figure 4 is the phase plane diagram of different pavement adhesion coefficient and the stable boundary provided by double-line method. From Figure 4, it can be noted that the higher the adhesion coefficient is, the stable area is larger, the stable boundary distance is bigger, and boundary slope basically remains unchanged . 

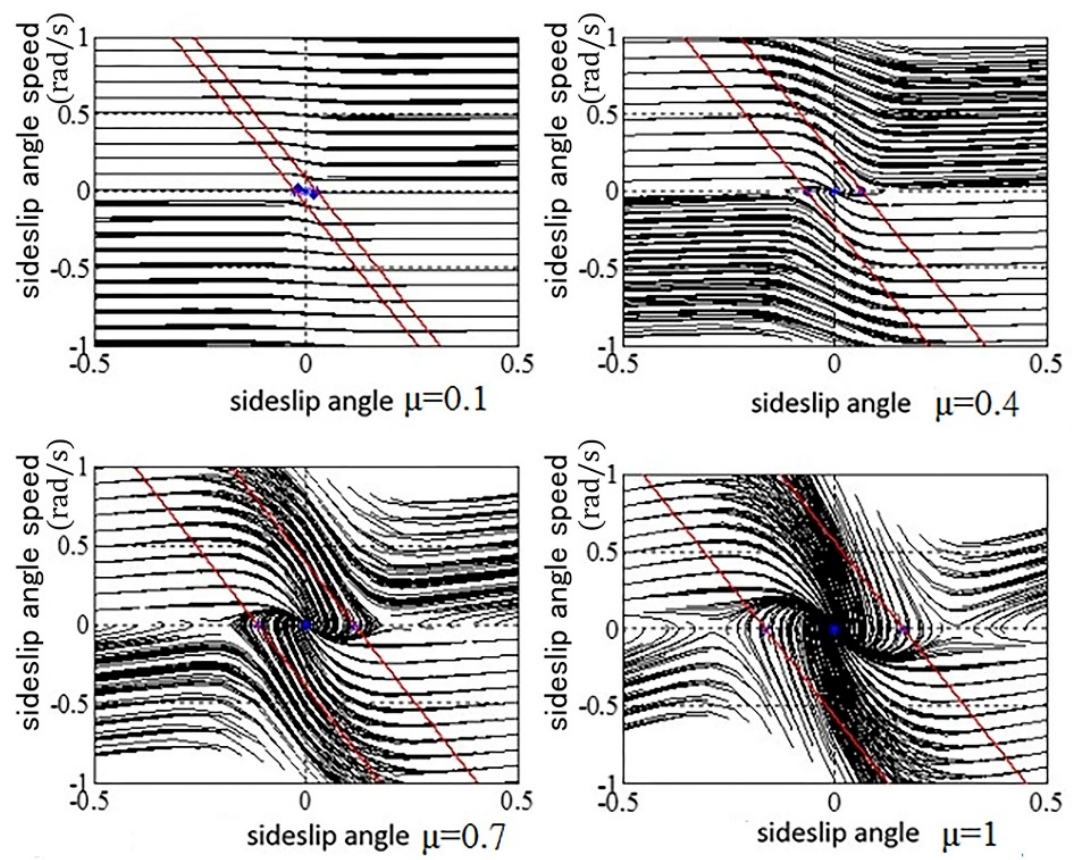

Fig. 4 Influence of adhesion coefficient on stability boundary

Figure 5 is the phase plane diagram of stable boundary at different speeds. Since under this condition, vehicle is in the stable state all the time, the influence of speed on the stable boundary is ignored.
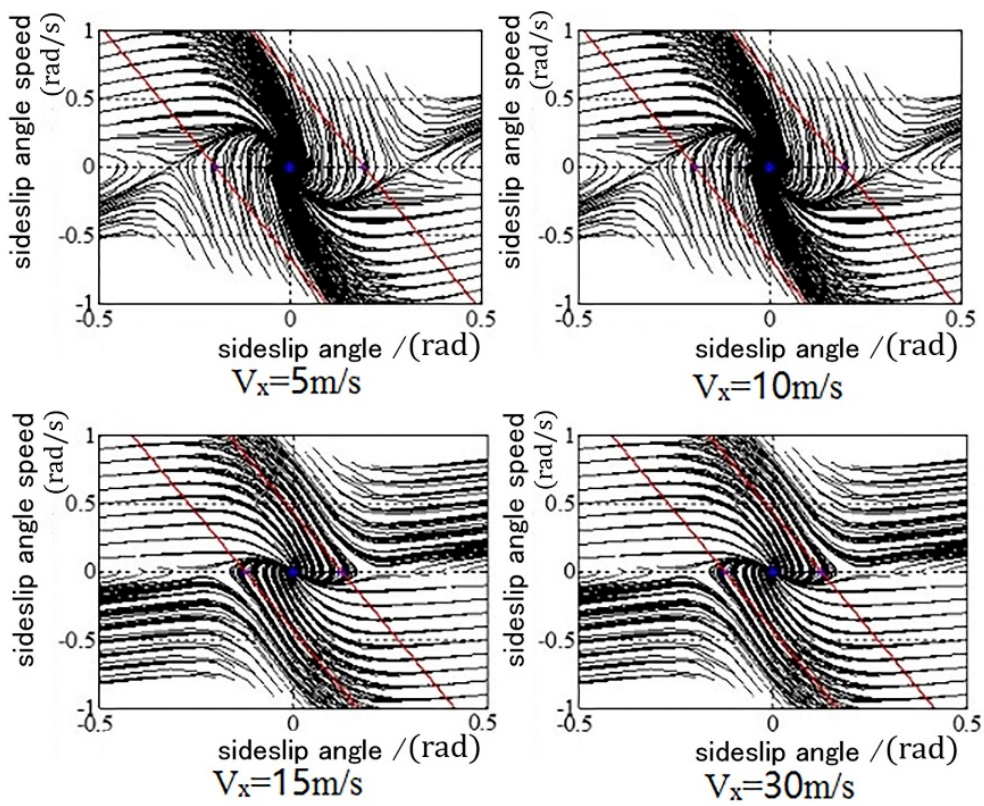

Fig. 5 Influence of speed on stability boundary

Figure 6 shows the phase plane of stable boundary at different front wheel turning angles. From Figure 6, it is obvious that when front wheel turning angle increases, the stable area moves to the right along the axis $\beta$; the ratio of displacement distance and front wheel turning angle are almost constant and stable area is basically unchanged. Moreover, the slope of the 
left straight line decreases with the increase of front wheel turning angle and the stable area in the second quadrant increases. Changes indicate that the front wheel turning angle affects the distribution of the stable area and the shape of a stable boundary.
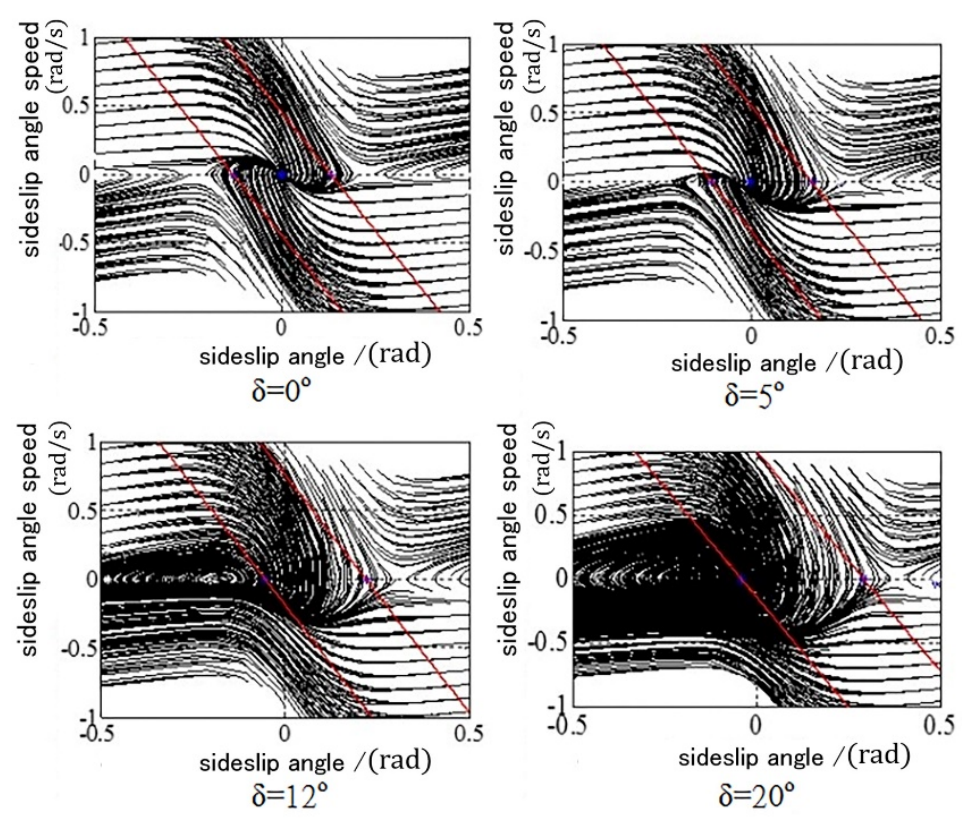

Fig. 6 Influence of front wheel angle on Stability Boundary

Based on the above analysis, the stable boundary with different pavement adhesion coefficient and front wheel turning angles is established. From Figure 7, it can be observed that doubleline method based on the conventional guidelines is used to divide the boundary. Basically, the stable area can be displayed, but is different from the actual hyperbolic boundary shown in Figure 7. The stable area provided by the double-line method is smaller and control system emits more fault signals. Therefore, three-fold lines are adopted to simplify the hyperbolic stable boundary on the $d \beta-\beta$ phase plane. Stable boundary schematic diagram after being simplified is shown in Figure 7. 


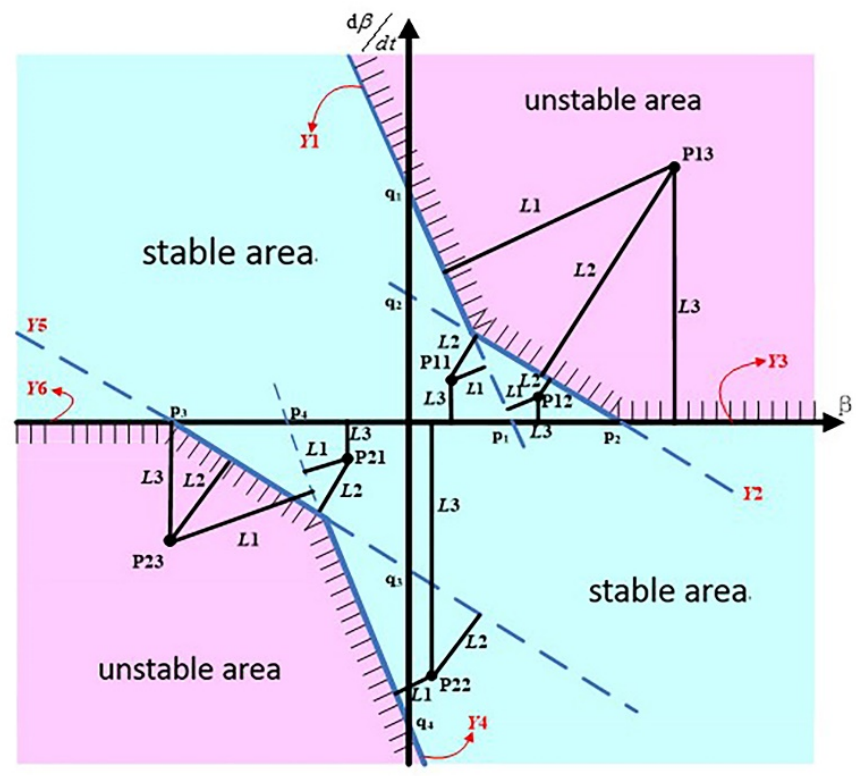

Fig. 7 Stable boundary with three-fold lines

In comparison with double-line method, fold line method is better. Fold lines can display more exactly the hyperbolic border, improving the accuracy and making it easier to express. In Figure 7, the distance from $Y 1$ to $Y 6$ is the stable boundary line; where $Y 3$ and $Y 6$ are horizontal axes. It is necessary to calculate the intercept from $p 1$ to $p 4$ and the distance from $q 1$ to $q 4$. According to the actual trajectory trend of a stable area, the slope of a certain proportion is increased or narrowed in such a way to form the approximate boundaries. The intercepts are calculated on these bases. Different pavement adhesion coefficient and front wheel turning angles correspond to different intercepts. The two intercept groups are compared and the final stable area is obtained according to the conventional principle.

\section{STABILITY CRITERION}

In Figure 7, the stable area is within the fold line; the part outside the fold line is unstable area. Different motion states correspond to P11, P12, P13, etc. After determining the stable boundaries for the positions of track points in the phase plane, they are referred to as the distance of three stable boundaries. The distance between track points and three stable boundaries (lope from the high to low) is calculated and respectively expressed as $L 1, L 2$ and L3. $L 3$ can be marked as $d \beta$ (ordinate of track point). The values of $L 1$ and $L 2$ are determined according to following criterion; when the distance line segment is between the corresponding line and the origin, distance value is negative and when it is outside the corresponding line and the origin, the distance value is positive.

$d \beta-\beta$ phase plane's stable boundaries can be expressed with intercept expression equation, as follows:

$$
\frac{\beta}{p_{j}}+\frac{d \beta}{q_{j}}=1
$$


In Equation (6), $j$ equals 1, 2, 3, 4, which corresponds to the intercepts $Y 1, Y 2, Y 3, Y 4$. According to the equation, the distance between the track point and the stable boundary is derived, as follows:

$$
L=\frac{p_{j} q_{j}}{\sqrt{p_{j}^{2}+q_{j}^{2}}}\left(\frac{\beta}{p_{j}}+\frac{d \beta}{q_{j}}-1\right)
$$

According to the above distance-related standards, if the smallest value of the three distance values for each track point is positive, the locus is in the unstable area and vice versa if the value is negative, the locus is in the stable area.

According to the above analysis, vehicle stability state criterion is derived as shown in Eq. (8):

$$
L_{m}=\min (L 1, L 2, d \beta)
$$

\section{SIMULATION}

To establish stability criterion, it is necessary to obtain information such as longitudinal speed, pavement adhesion coefficient and vehicle sideslip angle. These cannot be obtained by the sensor since its data collection accuracy is not able to meet the standards. If a sensor is used to collect data, operators need to make the appropriate state evaluation and use the observer. For this part, the author doesn't provide details. The proposed stability criterion is established in the Matlab/Simulink, integrated in yaw moment control model, combined with the vehicle model in Carsim, then the simulations of the control system are conducted.

\subsection{SIMULATION OF STABLE BOUNDARY}

For the set adhesion coefficient 0.2 , speed of $100 \mathrm{~km} / \mathrm{h}$ and small-angle sinusoidal input of the steering wheel, the simulation results are shown in Figure 8. Under these conditions, lateral acceleration speed is smaller; yaw rate is mostly consistent with the input trend of the steering wheel, vehicle is stable so its sideslip angle is within the stable area all the time.

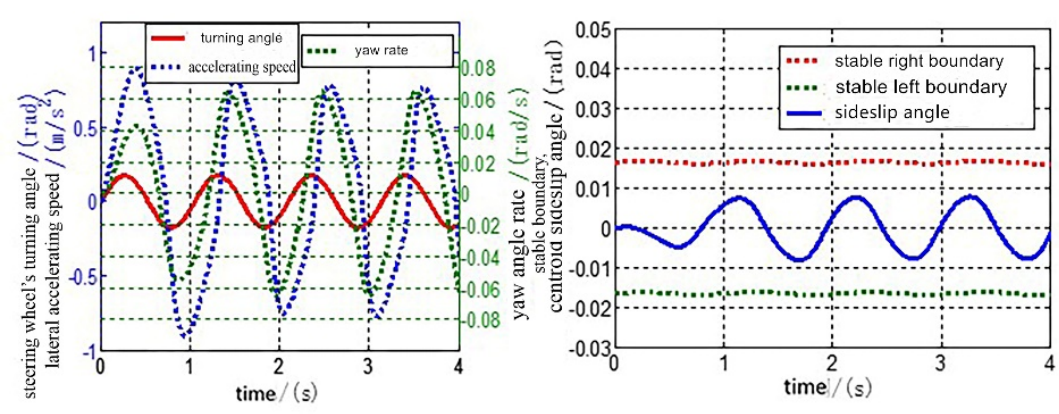

Fig. 8. Stable state simulation

For the set adhesion coefficient 0.2 , speed of $100 \mathrm{~km} / \mathrm{h}$ and big-angle sinusoidal input of the steering wheel, the simulation results are shown in Figure 9. From Figure 9, stems that the yaw rate lags behind the input of the steering wheel; vehicle is unstable due to the changes of lateral acceleration speed and vehicle sideslip angle is outside the stable boundary. 

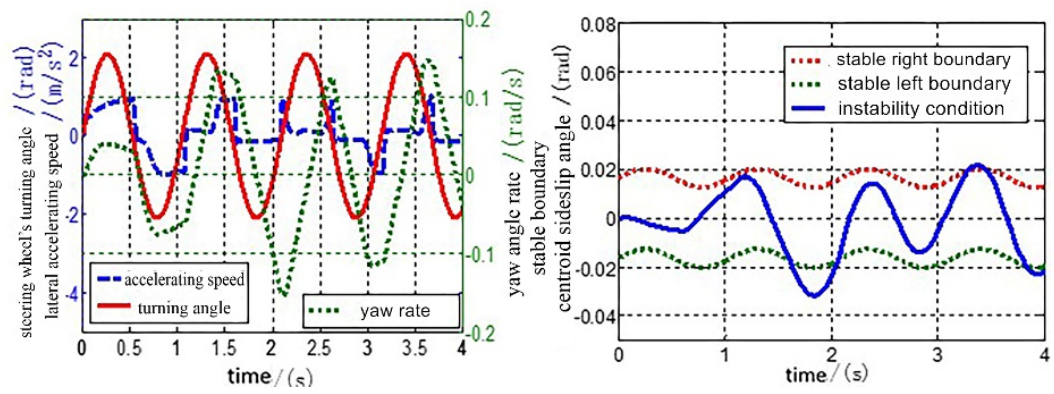

Fig. 9. Unstable state simulation

The stable and unstable state simulations are run on the pavement with adhesion coefficient 0.8 and sideslip angle, and changes of stable boundary are shown in Figure 10. Based on the simulation results, it is clear that the stable boundary of phase plane derived by the three-fold line method is effective.
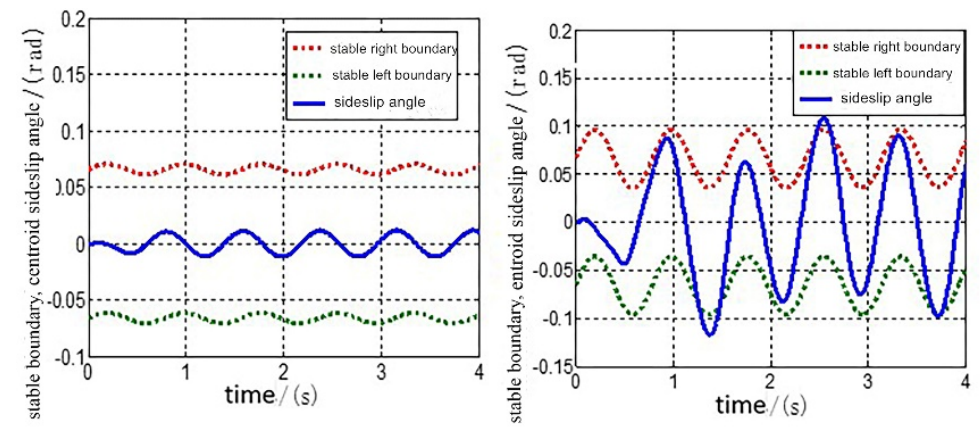

Fig. 10. Simulation under high adhesion coefficient

\subsection{SIMULATION OF CONTROL SYSTEM}

\subsubsection{STEERING AND STOPPING CONDITIONS}

FMVSS126 are the test standards for ESC stipulated by the American Highway Traffic Safety Administration. The test includes slowly increasing steer (SIS) and sine with dwell (SWD). Based on the above working conditions, control effects are tested. Firstly, simulations of SIS are run. From the simulation results stems that when the lateral acceleration speed is $0.3 \mathrm{~g}$, steering wheel's turning angle is $33.6^{\circ}$. Then, simulations of SWD are conducted. In this test, pavement adhesion coefficient is 0.6 ; the initial speed is $80 \mathrm{~km} / \mathrm{h}$; steering wheel's input which is its turning increases from $1.5 \mathrm{~A}$ to $6.5 \mathrm{~A}$, with the increase of $0.5 \mathrm{~A}$ each time. Simulation results are shown in Figure 11. From this Figure, it can be read that changes of yaw rate under the control of yaw moment and vehicle sideslip angle are consistent with the input of turning angle of steering wheel. What always converges is that the vehicle is unstable at the input value of steering wheel is $4.5 \mathrm{~A}$ if yaw moment is not applied. This indicates that the control system based on the $d \beta-\beta$ phase plane stability criterion can improve significantly vehicle stability. 

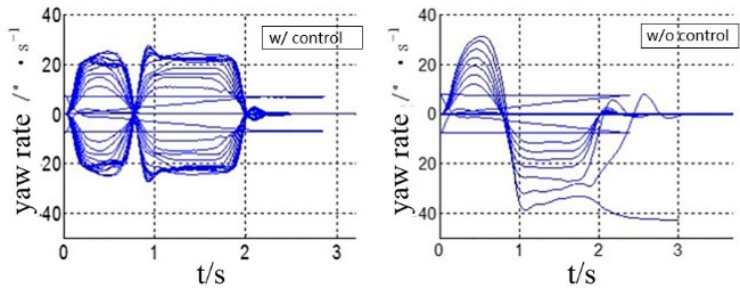

(a) Comparison of yaw rate speed
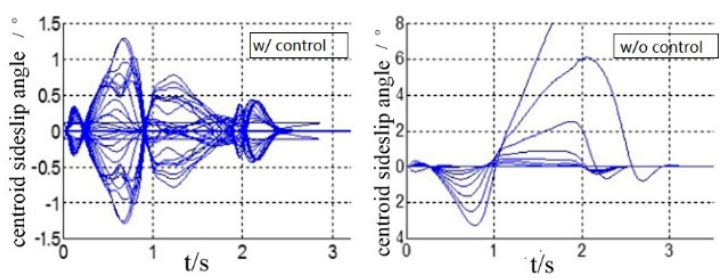

(b) Comparison of vehicle sideslip angle
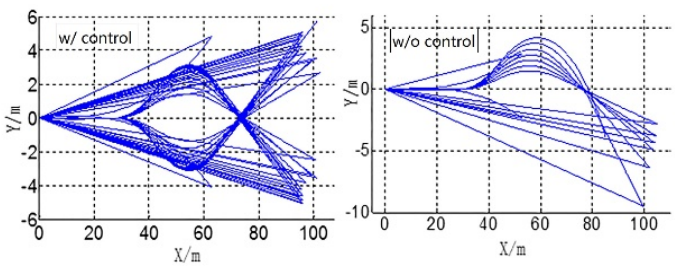

(c) Comparison of vehicle movement track
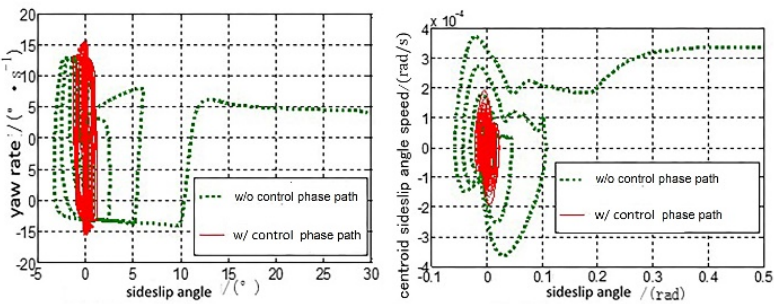

(d) Comparison of phase plane

Fig. 11 Control system simulation

The yaw rate decline and lateral displacement are calculated, the simulation results are evaluated and shown in Table 3. When starting the control system, yaw rate $1 \mathrm{~s}$ after the input is less than $35 \%$ of peak and after $1.75 \mathrm{~s}$, it is less than $20 \%$ of peak. Yaw rate decline is in line with the lateral stability indexes. Lateral displacement is not changed with the increase of steering angle. All values are higher than $1.83 \mathrm{~m}$, which means that actual turning operations meet the standards. When closing the control system, the vehicle is out of control at the turning angle higher than $4 A$, and the yaw rate is in divergent state. 
Table 3. Comparison of stability assessment

\begin{tabular}{|c|c|c|c|c|c|c|c|c|}
\hline \multirow[t]{2}{*}{$\delta w$} & \multicolumn{2}{|c|}{$\dot{\psi}_{\text {peak }} /(\mathrm{deg} / \mathrm{s})$} & \multicolumn{2}{|c|}{$\begin{array}{c}\text { Lateral } \\
\text { displacement } / \mathrm{m}\end{array}$} & \multicolumn{2}{|c|}{$\frac{\dot{\psi}_{t_{0}+1}}{\dot{\psi}_{\text {peak }}}$} & \multicolumn{2}{|c|}{$\frac{\dot{\psi}_{t_{0}+1.75}}{\dot{\psi}_{\text {peak }}}$} \\
\hline & Start & Close & Start & Close & Start & Close & Start & Close \\
\hline $1.5 \mathrm{~A}$ & -11.98 & -11.98 & - & - & -0.0912 & -0.0912 & -0.0331 & -0.0331 \\
\hline $2 A$ & -13.78 & -17.93 & - & - & -0.2469 & -0.1369 & -0.0278 & -0.0278 \\
\hline $2.5 \mathrm{~A}$ & -16.61 & -20.98 & - & - & -0.4532 & -0.1715 & -0.0429 & -0.0429 \\
\hline $3 \mathrm{~A}$ & -19.02 & -26.96 & - & - & -0.6338 & -0.2336 & -0.0445 & -0.0445 \\
\hline $3.5 \mathrm{~A}$ & -22.05 & -32.52 & - & - & -0.5739 & 4.7047 & -0.1143 & -0.1400 \\
\hline $4 A$ & -23.65 & -43.03 & - & - & -1.145 & 98.42 & -0.7937 & 98.42 \\
\hline $4.5 \mathrm{~A}$ & -23.79 & & - & & -1.339 & & -1.1003 & \\
\hline $5 \mathrm{~A}$ & -23.31 & & - & & -0.8783 & & -0.5629 & \\
\hline $5.5 \mathrm{~A}$ & -23.43 & unstable & 3.19 & unstable & -1.8433 & unstable & -1.3227 & unstable \\
\hline $6 A$ & -24.97 & & 3.21 & & -2.0477 & & -1.7727 & \\
\hline $6.5 \mathrm{~A}$ & -25.99 & & 3.31 & & -1.3385 & & -0.8242 & \\
\hline $270^{\circ}$ & -26.66 & & 3.33 & & -1.4901 & & -1.0933 & \\
\hline
\end{tabular}

\subsubsection{DOUBLE LANE CONDITION}

Simulations of ISO 3888-2 brake DLC condition are run with adhesion coefficient of 0.5 and initial speed of $50 \mathrm{~km} / \mathrm{h}$.

Simulation results are shown in Figure 12. From the comparisons of vehicle tracks in Figure 12 , it stems that the double lane condition is completed when starting or closing the yaw moment control system. The motion of the vehicle started by the yaw moment control system is closer to the standard lane track. If the stability control system is not started, the vehicle's turning angle can't meet the demands so the turning angle of steering wheel must be bigger in order to complete the double lane condition which results in the increase of driving burden. From the comparison of the results, it is obvious that when the vehicle is stable, the yaw rate can reach the expected or ideal value improving vehicle control performance. 

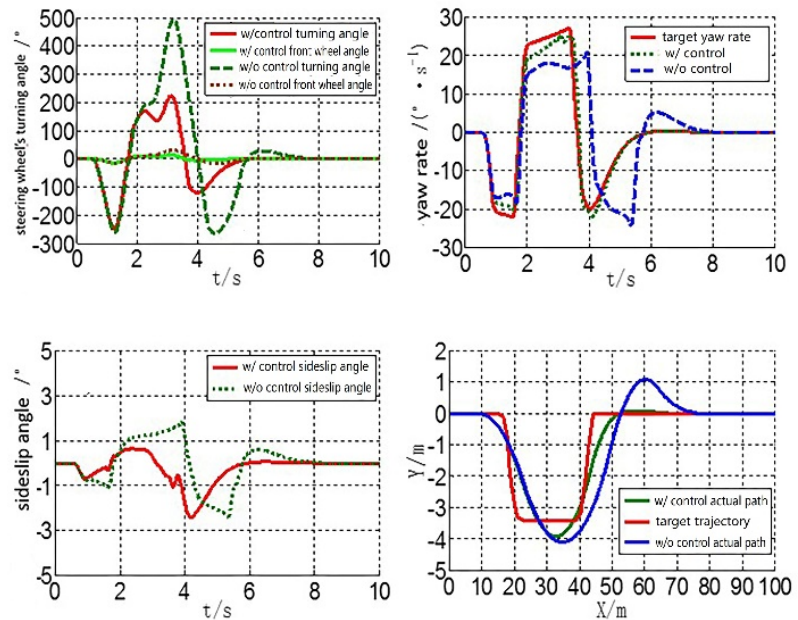

Fig. 12 Double lane change simulation results comparison

\section{CONCLUSION}

In this paper, phase plane method is used as a tool to analyse the changes of vehicle sideslip angle, vehicle sideslip angle speed phase plane and stable boundaries. Since the stable area determined by the double-line method is obviously smaller than the actual stable area, threefold line method is proposed to simplify the curve stability boundary, and appropriate stability criterion is established. Stability criterion and the yaw moment control system simulations verify the effects of the stability criterion while the simulation results provide references for the development and improvement of vehicle stability control system.

\section{ACKNOWLEDGMENTS}

This work is supported by National Natural Science Foundation of Jilin (No. 20150101037JC).

\section{REFERENCE}

[1] M. Takuya, R. Pongsathorn and N. Masao, Direct Yaw-moment Control Adapted to Driver Behavior Recognition [C], SICE-ICASE International Joint Conference, pp. 534-539, 2006. https://doi.org/10.1109/SICE.2006.315542

[2] C. Song, F. Xiao and S. Liu, State estimation of electric vehicle with in-wheel motors based on UKF, Journal of Jilin University (Engineering and Technology Edition), Vol. 46, No. 2, pp. 222-229, 2016. https://doi.org/10.13229/j.cnki.jdxbgxb201602001

[3] A. Zanten, Bosch ESP Systems: 5 Years of Experience, SAE 2000 Automotive Dynamics \& Stability Conference, 2000. https://doi.org/10.4271/2000-01-1633

[4] K. Guo, Vehicle handling dynamics, Jilin Science and Technology Press, 1991.

[5] Y. Luo and E. Lai, A research on vehicle stability judgment based on energy method, Automotive Engineering, Vol. 36, No. 12, pp. 1534-1538, 2014. 
[6] H. Pacejka, Non-linearities in Road Vehicle Dynamics, Vehicle System Dynamics, Vol. 15, No. 5, pp. 237-257, 1986.

https://doi.org/10.1080/00423118608968854

[7] S. Inagaki, I. Kushiro and M. Yamamoto, Analysis on vehicle stability in critical cornering using phase-plane method, JSAE Review, Vol. 16, No. 2, pp. 216-216, 1995.

https://doi.org/10.1016/0389-4304(95)94930-L

[8] H. Sacks and M. Singh, Automobile Stability-A Study of the Domain of Attraction, Vehicle System Dynamics, Vol. 6, No. 2, pp. 169-177, 1977.

https://doi.org/10.1080/00423117708968531

[9] M. Yamamoto, K. Koibuchi and Y. Fukada, Vehicle stability control in limit cornering by active brake, JSAE Review, Vol. 16, No. 3, pp. 323-323, 1995.

https://doi.org/10.1016/0389-4304(95)95150-S

[10] C. Zhang, Q. Xia and L. He, A Study on the Influence of Sideslip Angle at Mass Center on Vehicle Stability, Automotive Engineering, Vol. 33, No. 4, pp. 277-282, 2011.

[11] Z. Yu, B. Leng and L. Xiong, Vehicle Sideslip Angle and Yaw Rate Joint Criterion for Vehicle Stability Control, Journal of Tongji University (Nature Science), Vol. 43, No. 12, pp. 1841-1849, 2015. https://doi.org/10.11908/j.issn.0253-374x.2015.12.012

[12] L. Xiong, T. Qu and Y. Feng, Stability Criterion for the Vehicle under Critical Driving Situation, Journal of Mechanical Engineering, Vol. 51, No. 10, pp. 103-111, 2015. https://doi.org/10.3901/JME.2015.10.103

[13] F. Liu, L. Xiong and L. Deng, Vehicle Stability Criterion Based on Phase Plane Method, Journal of South China University of Technology (Nature Science Edition), Vol. 42, No. 11, pp. 63-70, 2014. https://doi.org/10.3969/j.issn.1000-565X.2014.11.010

[14] H. Dugoff and B. Brown, Measurement of Tire Shear Forces, SAE Technical Paper, No. 700092, pp. 316-324, 1970. https://doi.org/10.4271/700092

[15] H. Dugoff, P. Fancher and L. Segel, An Analysis of Tire Traction Properties and Their Influence on Vehicle Dynamic Performance, SAE Transactions, No. 700377, pp. 12191243, 1970. https://doi.org/10.4271/700377

[16] N. Ding and S. Taheri, A Modified Dugoff Tire Model for Combined-slip Forces, Tire Science and Technology, Vol. 38, No. 3, pp. 228-244, 2010.

https://doi.org/10.2346/1.3481696

[17] D. Wang and K. Guo, Simulation study on vehicle dynamics stability control, Automotive Technology, Vol. 11, No. 2, pp. 8-10, 1992. 